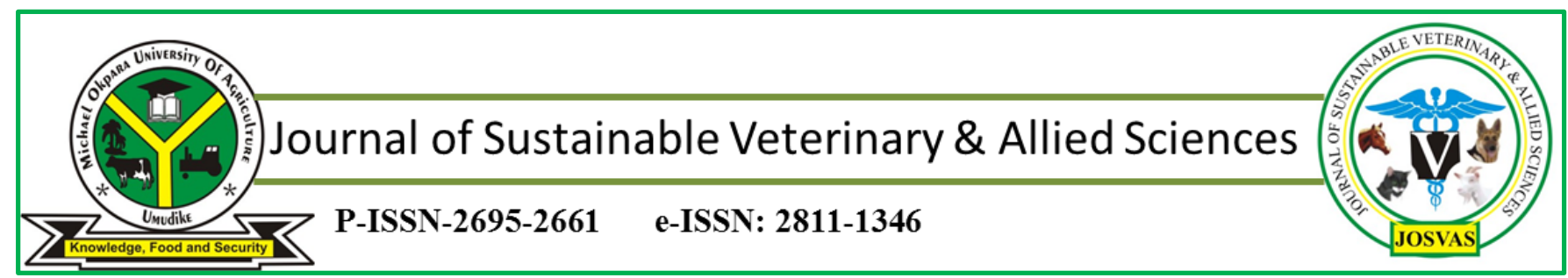

JoSVAS 2021, Vol 1, Issue 2:178-181

C2021 College of Veterinary Medicine,

Michael Okpara University of Agriculture, Umudike, Nigeria

Case Report

\title{
Unilateral uterine torsion in a preparturient Boer Bull bitch resolved by caesarian section and unilateral cornuectomy
}

\author{
${ }^{1 *}$ Raheem, K.A., ${ }^{2}$ Njoku, U. N., ${ }^{2}$ Ukweni C. P., ${ }^{2}$ Jeremiah K.T., ${ }^{1}$ Ani, N.V.,
}

${ }^{1}$ Odirichukwu, E.O. \& ${ }^{1}$ R.O. Ukaha ${ }^{1}$ Department of Theriogenology, ${ }^{2}$ Department of Surgery \& Radiology, Michael Okpara University of Agriculture, Umudike,

*Corresponding author: ka.raheem@mouau.edu.ng;+239031310693

\begin{abstract}
Uterine torsion is a rare finding in dogs and most often presented with a life-threatening condition. We hereby report a case of unilateral uterine torsion in a three-year old, pregnant bitch, two days to its expected date of delivery. The bitch became anorexic about two days prior to presentation and lumps of blood from vulva were found inside its kernel on the morning of presentation. The physiological parameters, except the rectal temperature $(38.70 \mathrm{C})$, were all outside the normal range for dogs. Haematology investigation revealed a packed cell volume (PCV) of $20 \%$ and hence, the need for haemaccel® administration. Digital evaluation of the birth canal with the middle finger only revealed a foetus with normal anterior presentation but no cervical dilation. Ultrasonography showed two live foetuses at the pelvic region of the bitch. Caesarean section was conducte d according to standard technique to save the two puppies in the left contralateral horn. The right uterine horn was twisted along its longitudinal axis over $360^{\circ}$ in a clockwise direction from inter-cornual junction. It had two dead foetuses and was filled with over 5 litres of clotted blood/dark fluid with a lot of necrotic tissue. The dead foetuses were removed and the right horn was excised after due consultation with the owner. The surgical incisions were closed according to standard techniques. Our timely intervention to do caesarean section and right unilateral cornuectomy saved the bitch and the two foetuses in the contralateral intact horn and partially preserved the reproductive potential of the bitch.
\end{abstract}

Keywords: Caesarean section, dystocia, unilateral cornuectomy, uterine torsion.

\section{INTRODUCTION}

Uterine torsion is an obstetrical condition involving the twisting of uterus or one of the uterine horns along its longitudinal axis of more than $45^{\circ}$ (Parkinson et al., 2019). The condition is rare in dog possibly because dog is a litter bearing species and the presence of foetuses in the two horns seems to stabilise the position of the uterus and minimise uterine torsion unlike in monotocous species like ruminant. Uterine torsion is mostly associated with gravid uterus and occurs usually during the third trimester with higher incidence during parturition. Causes of uterine torsion include hyper contraction of the uterus and excessive weight of one horn than the other such that delays the contraction in the heavier horn compared to the lighter uterine horn (Noakes et al., 2009). Other causes include excessive exercise that involves high jumping or rolling behaviour
, premature uterine contraction in late pregnancy, high foetal physical activities, partial abortion, hereditary weakness or compromise of broad and ovarian ligaments as supporting structures to the uterus. The entire aforementioned are associated with mobility of the uterus and inducible by trauma such as falling from a height (Kuroda et al., 2017).

Uterine torsion in bitch may be unilateral or bilateral and also occurs in gravid or non-gravid bitch. Bilateral uterine torsion has been reported in a non-gravid old bitch (Shull et al., 1978) possibly due to weakness of the broad ligament which supports and hold the uterus in situ. The degree of torsion may vary from $180^{\circ}$ to $270^{\circ}$ (Kumru et al., 2011) while few cases have reported over $360^{\circ}$ torsion in dog especially from the ovarian attachment (Kodie et al., 2019). It may also be congenitally induced as was found with congenital bilateral segmental aplasia of the uterine horn in a Pomeranian bitch (Nakamura et al., 2012). Sometimes, the condition may be 
secondary to an inflammatory endometrial polyp in the bitch (Chambers et al., 2011) or haematometra and cystic endometrial hyperplasia in a bitch (Barrand, 2009).

Uterine torsion is always a life threatening to both the foetus in gravid uterus and/or the dam in non-gravid uterus. Therefore, an immediate surgery is required to correct the torsion and expel the foetus in gravid animals.

\section{CASE HISTORY}

A 3-year old pregnant Boer bull bitch was referred to the Veterinary Teaching Hospital, Michael Okpara University of Agriculture, Umudike, Nigeria from Animal World, Umuahia. There was a complaint of bloody vulva discharge and loss of appetite for the past two days ago. History revealed this was the third pregnancy and the bitch was expected to whelp within the next 48 hours. The earlier two parturitions were not eventful and required no assistance.

\section{CLINICAL EXAMINATION}

The physiological parameters, except the rectal temperature $\left(38.7^{\circ} \mathrm{C}\right)$, were all outside the normal range for dogs. The heart, pulse and respiratory rates were $180 \mathrm{bpm}, 170 \mathrm{bpm}$ and $56 \mathrm{cpm}$ respectively. The dog was stable and the mucous membrane was pale with capillary refill time of 3 secs. Blood sample was taken for haemogram and the PVC was $20 \%$ (normal 35-57\%).

Digital palpation of the birth canal revealed an un-dilated cervix as only one finger could pass through the cervix. The head of a foetus was touched and signs of live were observed. The foetus was in longitudinal anterior presentation. Further diagnosis with the use of ultrasound was indicated. Ultrasonography revealed presence of two foetuses in the uterus at the pelvic region. The heart beats of two foetuses were picked by the ultrasound machine (Figure 1). Caesarean section was recommended and the animal was moved immediately to the surgical theatre.

\section{TREATMENT}

\section{CAESAREAN SECTION/UNILATERAL CORNUECTOMY}

The ventral abdomen was clipped, scrubbed with povidone iodine, and the patient was appropriately draped for the surgery and positioned on dorsal recumbency. Due to the low PCV and the unavailability of blood for transfusion to stabilize the bitch, a colloid (Haemaccel®, India) was infused at the rate of $10 \mathrm{mg} / \mathrm{kg} / \mathrm{hr}$ via the cephalic vein through a preplaced 21 gauge scalp vein set. Xylazine was used for preanaesthetic medication and was administered at a dose of $0.7 \mathrm{mg} / \mathrm{kg}$ i.m. while anaesthesia was induced with ketamine at a dose of $10 \mathrm{mg} / \mathrm{kg}$ through the intramuscular route and maintained using same ketamine at $7 \mathrm{mg} / \mathrm{kg}$ given intramuscularly.
The caesarean section was performed as described in an earlier study by Kodie et al., ( 2019). Briefly, a ventral midline incision was made from the umbilicus caudally towards the pubis. The left gravid uterine horn was identified and exteriorized. An incision was made on the uterine horn, over a presenting foetus, and the foetus was delivered with its placenta. Foetal membranes were removed and respiration was initiated. The umbilicus of the puppy was clamped and the puppy was towelled and transferred to a padded, heated box. Another puppy was milked to the hysterotomy incision and delivered in the same manner like the first set of puppies. The right gravid uterine horn was very big and stuck between the stomach, spleen and jejenum and could not be exteriorized prior to removal of its content. An incision was made on it and a large volume of clotted blood/dark fluid of about 5 litters was evacuated before the two dead foetuses could be removed. On exteriorization, this horn was observed to have twisted over $360^{\circ}$ from the intercornual junction along its longitudinal axis in a clockwise direction (Figure 2). The twisting was corrected and close examination of the right uterine revealed extensive widespread necrosis of the uterine tissues. A decision to amputate the right uterine horn was taken after due consultation with the owner. The incision on the left uterine horn was closed using size 1 catgut applied in a Lembert suture pattern. Thereafter, the inter-cornual junction was transfixed using size 1 catgut and viable blood vessels in the broad ligament were ligated. The necrotic right uterine horn was severed from the body of the uterus at a point distal from the point of transfixation and the horn removed. The peritoneum and abdominal muscles were sutured individually using catgut (size 1) in a interlock suture pattern. The subcutaneous tissue was closed using catgut (size 1) in a subcutucular suture pattern, while the skin was closed using silk (size 0) applied in horizontal mattress pattern.

Post operatively, the dog was administered ceftraizone at 25 $\mathrm{mg} / \mathrm{kg}$ (intravenously) and diclofenac at $0.3 \mathrm{mg} / \mathrm{kg}$ (intramuscularly) once daily for 10 and 3 days respectively. The two puppies (Figure 3 ) survived and were introduced to the bitch about two hours after recovery.

\section{DISCUSSION}

Obstetrical interventions in dystocia include manipulation of the foetus to correct for abnormal foetal disposition, traction, chemotherapeutic and caesarean section (Adeyeye 2017). In this particular case, non-dilation of the cervix made the first three listed treatments of dystocia practically impossible.

Chemotherapeutic treatment involves used of ecbolic such as oxytocin to initiates uterine contraction and subsequent expulsion of foetus (Johnson, 2004). This method was not used in this present case because there was practically no 


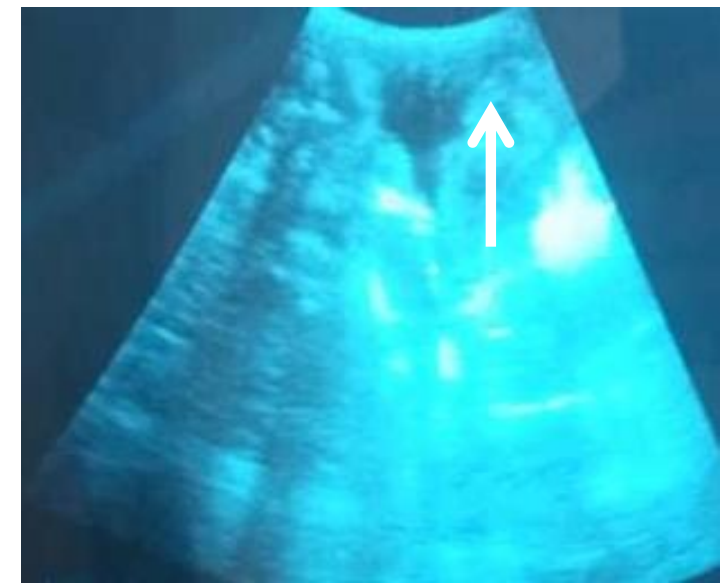

Figure 1. Ultrasonography showing live foetus heart beat picked at the white arrow

Perhaps, erroneous administration of oxytocin in the present condition could have led to uterine rupture. An indiscriminate use of oxytocin was reported earlier as cause of uterine torsion in a pregnant Labrador bitch (Kodie et al., 2019). The primary cause of uterine torsion in this case remains uncertain. Though there were no signs of labour as at the time of presentation, it was possible the bitch came into labour during the night and the hyper contraction of the uterus or hyperactivity of the foetuses culminated into uterine torsion.

The primary aims of obstetrical intervention are to safeguard the life of the foetus and the dam and preserve the reproductive potential of the dam (Adeyeye, 2017). In situations where a choice between the foetal life or dam is required, the life of the dam is preferred over the foetus. In the present case, our immediate intervention saved

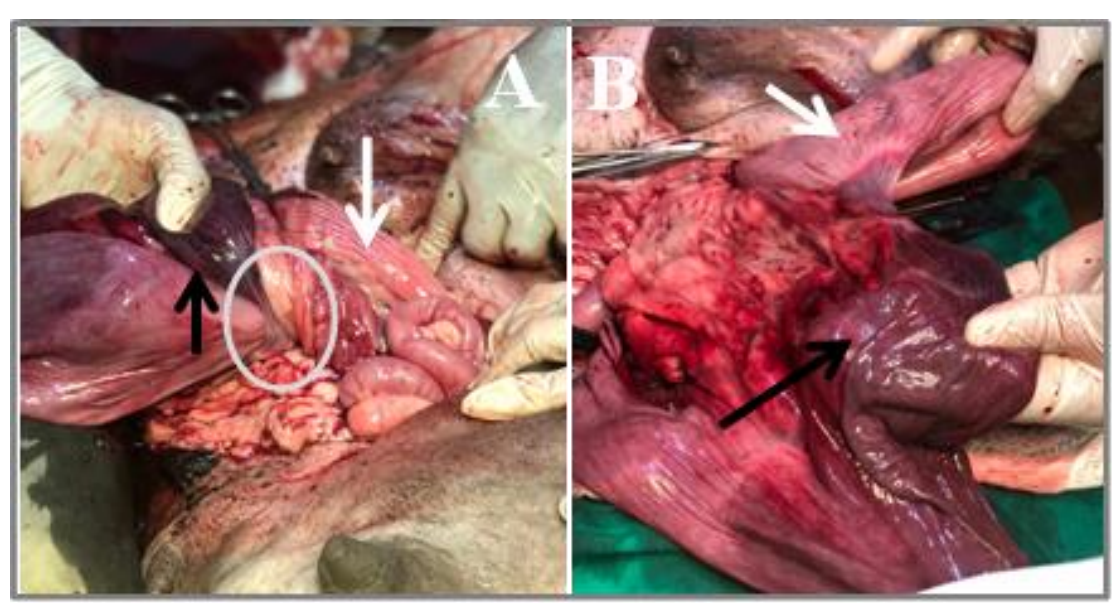

Figure II. Twisted right uterine horn (black arrow) before correction (A) and after correction (B) ischaemic compared to the normal left (white arrow) as well as region of twisting (circle)

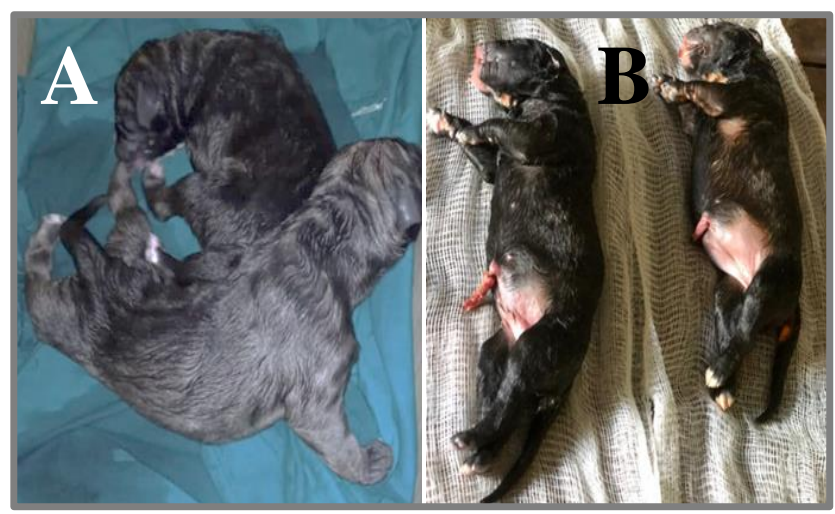

Figure III. Image of two live (A) and dead (B) foetuses post caesarean section

cervical dilation. Therefore, uterine contraction that is not preceded by cervical dilation will not resolve a dystocia case. the two foetuses in the contralateral left intact horn as well as the bitch. Otherwise, nonappetite and continuous loss of blood by the bitch due to rupture of blood vessels were eventually going to affect the metabolic need of the live foetus provided by the bitch. Foetal death in both horns is imminent in uterine torsion possibly prior to the maternal death in an untimely intervention.

The foetuses on the twisted horn might have died of suffocation since the uterine vessels were twisted along with the uterine horn. In addition, the rupture of blood vessel into right uterine horn evidenced by about 5 litres of clotted blood/dark fluid was capable of compromising the function of placenta which is a communication network for exchange of gases and micronutrient between the mother and the foetus. The pathogenesis of this condition seemed to be clear. The first series of complication following uterine torsion is ischaemia and loss of blood supply to the uterus and then foetus leading to significant reduction in transport of micronutrient and gases from the mother to the foetus and ultimately foetal death (Johnston et al., (2001). The foetuses on the twisted horn died of suffocation since the uterine vessels were twisted along with the uterine horn. In addition, the rupture of blood vessel into right uterine horn evidenced by about 5 litres of clotted blood/dark fluid was capable of compromising the function of placenta as a communication network for exchange of gases and micronutrient between the mother and the foetus. Uterine torsion may also become complicated with other pathological conditions of abdominal organs such as spleen due to build up pressure within the associated supporting structures. Simultaneous torsion of uterine horn and spleen was reported in a pregnant two-year- 
Old crossbreed bitch (Martins-Bessa et al., 2020). In the present case, the twisted horn was actually stuck below the spleen and the stomach. Uterine torsion may be complicated by hypovolemic shock, peritoneal effusion and uterine rupture as well septic peritonitis The prognosis of aforementioned complications are very poor, however successful treatment of such cases have been reported (Ritt \& Fossum, 1997).

The treatment of choice for uterine torsion in non-pregnant animal is usually exploratory laparotomy (Misumi et al., 2000). In gravid horn, the treatment is caesarean section followed by unilateral cornuectomy depending on the degree of necrosis of the uterine tissue and the owner's preference in keeping reproductive potential of the animal. Otherwise, bilateral cornuectomy is conducted for permanent prevention of future occurrence. The dog handled in this present case is kept for breeding and the bitch whelped 13 puppies in the last pregnancy and not a single one died. Therefore, unilateral excision of twisted right uterine horn was done to preserve the reproductive capacity of the bitch. In addition, the good condition of the right uterine horn prompted this decision.

We envisaged the bitch will be able to conceive again as subsequent pregnancies after treatment of unilateral uterine torsion by partial hysterectomy have been reported (Kumru et al., 2011) despite the possibility of reduction in the litter birth weight. To prevent future occurrence of uterine torsion, pregnant bitch should be adequately monitored during the last three days prior to and/or after expected day of delivery. Such practice will reveal the possible cause of the dystocia for prompt and appropriate obstetrical intervention to be instituted in order to salvage the live of the pregnant bitch and her foetus (es). In conclusion, the outcome of this study demonstrates that the foetuses in the intact horn have high chances of survival compared to the foetus in the contralateral twisted horn, provided the obstetrical intervention is timely.

\section{REFERENCES}

Adeyeye, A. (2017). Parturition and Neonatal Care. In K. A. Raheem (Ed.), Introductory Theriogenology. (pp. 70-78). Ibadan: University Press PLC.

Barrand, K. R. (2009). Unilateral uterine torsion associated with haematometra and cystic endometrial hyperplasia in a bitch. Veterinary Record, 164(1), 19-20.

Chambers, B., Laksito, M., Long, F. \& Yates, G. (2011). Unilateral uterine torsion secondary to an inflammatory endometrial polyp in the bitch. Australian Veterinary Journal, 89(10), 380-384.

Jackson, P.G.G. (2004). Handbook of Veterinary Obstetrics,, edn 2nd, pp. 173-199. Edinburgh, UK: Saunders.

Johnston, S.D., Root, Kustritz M.V \& Olson, P.N.S. (2001). Canine parturitio: eutocia and dystocia. In: SD Johnston, MV Root Kustritz, PNS Olson, editors:
Canine and Feline Theriogenology. Philapdelphia, USA: W.B. Saunders; pp. 122-125.

Kodie, D. O., C.O. Oguntoye, N.S. Oyetayo \& Eyarefe, O. D. (2019). Unilateral uterine torsion with a nearcomplete rip of the affected right horn in a parturient German shepherd bitch. Sokoto Journal of Veterinary Sciences, 18(2), 108-113.

Kumru, I. H., Seyrek-Intas, K., Seyrek-Intas, D., Tek, H. B.\&Wehrend, A. (2011). Clinical case: Unilateral en bloc ovariocornuectomy as a treatment for uterine torsion in a bitch. Revue de Médecine Vétérinaire, 162(2), 76-78. .

Kuroda, K., Osaki, T., Harada, K., Yamashita, M., Murahata, Y., Azuma, K., Tsuka, T., Ito, N., Imagawa, T. \& Okamoto, Y. (2017). Uterine torsion in a full-term pregnant cat. JFMS open reports, 3(2), 2055116917726228-2055116917726228.

Martins-Bessa, A., Vieira, L., Machado, J., Almeida, M., Alves, T., Fachada, M. T., Pires, M. A., Alves, A. \& Dias, I. R. (2020). Simultaneous Torsion of a Uterine Horn and Spleen in a Pregnant Two-YearOld Crossbreed Bitch: A Case Report. Topics in Companion Animal Medicine, 41, 100459.

Misumi, K., Fujiki, M., Miura, N. \& Sakamoto, H. (2000). Uterine horn torsion in two non-gravid bitches. Journal of Small Animal Practice, 41 (10), 468-471.

Nakamura, K., Yamasaki, M., Osaki, T., Ohta, H., Sasaki, N., Aoshima, K., Kimura, T. \& Takiguchi, M. (2012). Bilateral segmental aplasia with unilateral uterine horn torsion in a Pomeranian bitch. Journal of the American Animal Hospital Association, 48(5), 327-330.

Noakes, D.E., T.J. Parkinson \& England, G.C. (2009) Veterinary reproduction and obstetrics. Edn 9th. China: Elsevier.

Parkinson, T., Vermunt, J. \& Noakes, D. (2019). Maternal Dystocia: Causes and Treatment. In T. P. G. E. DE Noakes (Ed.), Veterinary Reproduction and Obstetrics (pp. 236-249). Philadelphia: WB Saunders Co.

Ritt, M. G. \& Fossum, T. W. (1997). Successful treatment of uterine torsion and fetal retention in a postparturient Great Pyrenees bitch with septic peritonitis and prothrombotic complications. Journal of the American Animal Hospital Association, 33(6), 537 539.

Shull, R. M., Johnston, S. D., Johnston, G. R., Caywood, D. \& Stevens, J. B. (1978). Bilateral torsion of uterine horns in a nongravid bitch. Journal of the American Veterinary Medical Association, 172(5), 601-603.

Article history: Received: June 26, 2021, Revised: July 30, 2021, Arrented. Ausust 10 2071 